\title{
The potential role of associating liver partition and portal vein ligation in the treatment of colorectal liver metastases
}

\author{
Jan P. Jonas ${ }^{1,2}$, Henrik Petrowsky ${ }^{1}$ \\ ${ }^{1}$ Swiss HPB and Transplantation Center, Department of Surgery, University Hospital Zurich, Zurich, Switzerland; ${ }^{2}$ HPB Center, Department of \\ Surgery, Clinic Favoriten, Vienna, Austria \\ Correspondence to: Henrik Petrowsky, MD, FEBS (HPB), FACS. Swiss HPB and Transplantation Center Zurich, Department of Surgery and \\ Transplantation, University Hospital Zurich, 8091 Zurich, Switzerland. Email: Henrik.petrowsky@usz.ch. \\ Comment on: Glinka J, Ardiles V, Pekolj J, et al. The role of associating liver partition and portal vein ligation for staged hepatectomy in the \\ management of patients with colorectal liver metastasis. Hepatobiliary Surg Nutr 2020;9:694-704.
}

Submitted Oct 10, 2021. Accepted for publication Oct 25, 2021.

doi: $10.21037 / \mathrm{hbsn}-21-420$

View this article at: https://dx.doi.org/10.21037/hbsn-21-420

Life expectancy of patients with colorectal liver metastasis (CRLM) significantly improved over the last decades due to the induction of multimodal effective treatment strategies including perioperative chemotherapy and advanced surgical procedures. By conversion chemotherapy, previously unresectable CRLMs can be converted to a resectable situation and amenable to procedures such as associating liver partition and portal vein ligation (ALPPS) (1). A recent publication in $H B S N$ has extensively reviewed ALPPS as a concept in the treatment of CRLM. This review article by Glinka et al. (2) focuses on the evolvement of this novel procedure and describes in detail the gradual changes accompanying this procedure's evolvement since 2007. Further, the authors highlight several aspects crucial for the best possible outcome after this procedure including patient selection, future liver remnant, technical aspects, safety, morbidity, mortality and oncological results.

The authors highlighted that the first years after the introduction of ALPPS were marked by repeated concerns addressing the observed safety issues with high morbidity and mortality and uncertain oncological benefit of this major hepatectomy procedure (3). Historically, ALPPS was used to treat not exclusively CRLMs but primary liver tumors as well such as hepatocellular carcinoma (HCC) or cholangiocarcinoma (CCC). Meanwhile, the preferred indication for ALPPS gradually shifted towards CRLM (Figure 1) as these patients showed less morbidity and mortality rates compared to other liver cancer indications. Best perioperative outcome was observed in
CRLM patients younger than 60 years, no underlying liver disease and absence of postoperative red blood cell transfusion $(2,4)$. Concomitant liver disease such as cirrhosis or steatohepatitis can negatively affect postoperative regenerative capabilities of the liver, which is especially hindering in the ALPPS setting. Nonetheless, the review highlights on post-hepatectomy liver failure (PHLF), which is defined by the ISGLS criteria (5), as the leading cause of postoperative morbidity and mortality and can still occur in $16 \%$ to $30 \%$ in spite of sufficient future liver remnant (FLR) (6). The discordance between FLR volume and liver function suggests that calculation of FLR only is insufficient for determining the risk of PHLF. Therefore, the authors advocated for measuring FLR function (FLRF) in assisting patient selection and timing of stage 2 ALPPS. Many non-invasive methods and approaches have been proposed to assess FLRF; however, most of these tools have limitations. In this article, the authors especially review the modification of a biliary scintigraphy technique based on ${ }^{99 \mathrm{~m}} \mathrm{Tc}$-mebrofenin, which runs on computed tomography-single photon emission computed tomography (CT SPECT) imaging. The dynamic of the tracer can be measured and used for FLRF assessment before ALPPS stage 2. The functional data are overlapped with CT imaging (HIBA scan) and can correctly predict PHLF in $80 \%$ of patients as proposed by the Argentinian group (7). A recent systematic review summarized the FLRF tests and described their current value in modern HPB surgery (8). The conclusion of this review was that only preoperative 


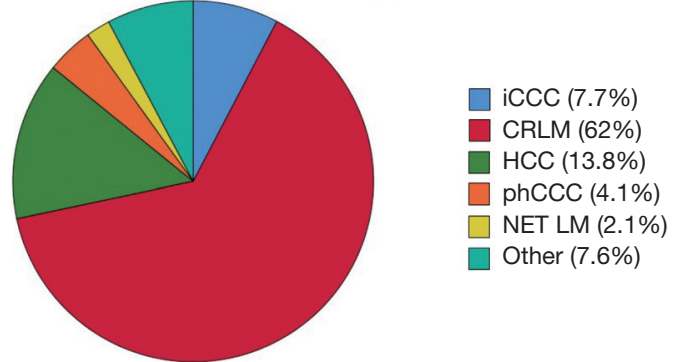

Figure 1 Indications for ALPPS according to the ALPPS Registry (2009-2021). Last data export January 2021 (available online: http://www.alpps.net/?q=registry). iCCC, intrahepatic cholangiocarcinoma; CRLM, colorectal liver metastases; HCC, hepatocellular carcinoma; phCCC, perihilar cholangiocarcinoma; NET LM, neuroendocrine liver metastases.

volumetric work-up of potential ALPPS patients is not adequate and complementary function tests are mandatory for correct patient selection. A promising approach to define FLRF is based on liver MRI with Gd-EOB-DTPA as a hepatocyte-specific contrast agent, which allows for simultaneous volumetric and functional assessment without the need for ionizing radiation. However, no clear and well validated guidelines with defined cut-offs exist on FLRF analysis to this date. Therefore, the expert HPB community should standardize FLRF cut-offs, which would allow cross comparability among centers. Nonetheless, there is growing evidence that both FLR volumetry and function assessment remain most crucial for surgery and should be thoroughly standardized with easy-to-use guidelines.

Until recently, classical two-stage hepatectomy (TSH) was considered to be the standard approach for colorectal disease with bilobar liver metastases. Resection rates (RR) of up to $60 \%$, good perioperative outcome and 5 -year overall survival up to $40 \%$ earned this approach considerable popularity. Accordingly, first available data on ALPPS outcome after its introduction considered this procedure as inferior compared to TSH in terms of survival, morbidity and mortality (9). Correspondingly, an early systematic review from 2017 concludes that ALPPS was significantly associated with a higher overall morbidity compared to TSH (10). Since then, ALPPS has undergone undoubtedly many modifications due to significant advances in operative techniques and safe patient selection (11). Here, the authors mentioned that the perioperative 90-day mortality of ALPPS was $16 \%$ in the years up to 2011 , which; however, gradually decreased over time to $3.8 \%$. Intriguingly, a systematic review which has been published recently in 2020 shows no significant differences in terms of overall morbidity and mortality between ALPPS and classical TSH and therefore reflects the gradual improvements of this technique (12). In addition, the only prospective, randomized controlled trial comparing ALPPS vs. TSH and its most recent follow-up analysis, published in 2021 (LIGRO trial, initiated in 2014), showed that morbidity and 90-day mortality were comparable between ALPPS and classical TSH procedures $(13,14)$. In terms of RR, ALPPS seems to achieve RR rates superior to those of classical TSH $(92 \%$ vs. $80 \%)$ in initially non-resectable CRLM patients $(13,14)$.

Usually, the classical ALPPS approach aims at cleaning the FLR, partially transect the parenchyma and ligate the right portal vein in a first step and, in a second step, is completed with a right trisectionectomy. The authors of the review describe several ALPPS variations, which have been introduced over time to cope with initial high perioperative morbidity and mortality of this surgical approach. According to the authors, instead of pedicle dissection followed by surgical trauma an endovascular approach can be used for portal vein embolization (PVE) with the aim to avoid hepatic manipulation. As highlighted in this article's section, many ways exist how to transect the parenchyma with a visible trend of low morbidity and mortality associated with decreased ALPPS invasiveness and therefore less surgical trauma $(11,15)$.

At time of publication of the review article by Glinka et al. (2), no long-term data on oncological outcome was available for patients who underwent ALPPS. Initial data from 2014 suggested that these patients had comparable short-term oncological outcome as classical TSH procedures with 12 -month disease-free survival of 59\% (4). Nevertheless, a corresponding article was published recently and is based on the international, multicentric, prospectively maintained ALPPS Registry database (NCT01924741), which has been established in 2012. Over a 10-year period, 510 patients were included in this publication with a median follow-up of 38 months and long-term oncological outcome was demonstrated. Here, the median overall and recurrencefree survival was 39 and 15 months (16). In comparison, the Scandinavian LIGRO trial, which is a randomized controlled trial comparing ALPPS vs. TSH, showed a similar trend with significantly better median overall survival favoring ALPPS over TSH (46 vs. 26 months, $\mathrm{P}=0.028$ ) (14). Furthermore, the ALPPS registry study showed that tumor biology [primary T4, right colon cancer, 
Kirsten rat sarcoma/proto-onogene (KRAS)] as well as response to chemotherapy were identified as independent oncological survival predictors. These findings suggest that meticulous patient selection based on tumor biology and chemotherapy response remains most crucial for optimal oncological outcome in previously unresectable patients treated with ALPPS.

In conclusion, a certain degree of controversy towards ALPPS remains today in many centers. However, over the last decade many efforts have led to a better understanding of patient selection, indications, technical feasibility and limitations. Therefore, ALPPS should be considered as a safe surgical approach in HPB centers with low morbidity and mortality, good oncological outcome and high patient safety.

\section{Acknowledgments}

Funding: None.

\section{Footnote}

Provenance and Peer Review: This article was commissioned by the editorial office, Hepatobiliary Surgery and Nutrition. The article did not undergo external peer review.

Conflicts of Interest: Both authors have completed the ICMJE uniform disclosure form (available at https://hbsn. amegroups.com/article/view/10.21037/hbsn-21-420/coif). Dr. HP serves as an unpaid editorial board member of Hepatobiliary Surgery and Nutrition. The other author has no conflicts of interest to declare.

Ethical Statement: The authors are accountable for all aspects of the work in ensuring that questions related to the accuracy or integrity of any part of the work are appropriately investigated and resolved.

Open Access Statement: This is an Open Access article distributed in accordance with the Creative Commons Attribution-NonCommercial-NoDerivs 4.0 International License (CC BY-NC-ND 4.0), which permits the noncommercial replication and distribution of the article with the strict proviso that no changes or edits are made and the original work is properly cited (including links to both the formal publication through the relevant DOI and the license). See: https://creativecommons.org/licenses/by-nc-nd/4.0/.

\section{References}

1. Petrowsky H, Fritsch R, Guckenberger M, et al. Modern therapeutic approaches for the treatment of malignant liver tumours. Nat Rev Gastroenterol Hepatol 2020;17:755-72.

2. Glinka J, Ardiles V, Pekolj J, et al. The role of associating liver partition and portal vein ligation for staged hepatectomy in the management of patients with colorectal liver metastasis. Hepatobiliary Surg Nutr 2020;9:694-704.

3. Schnitzbauer AA, Lang SA, Goessmann H, et al. Right portal vein ligation combined with in situ splitting induces rapid left lateral liver lobe hypertrophy enabling 2 -staged extended right hepatic resection in small-for-size settings. Ann Surg 2012;255:405-14.

4. Schadde E, Ardiles V, Robles-Campos R, et al. Early survival and safety of ALPPS: first report of the International ALPPS Registry. Ann Surg 2014;260:829-36; discussion 836-8.

5. Rahbari NN, Garden OJ, Padbury R, et al. Posthepatectomy liver failure: a definition and grading by the International Study Group of Liver Surgery (ISGLS). Surgery 2011;149:713-24.

6. Schadde E, Raptis DA, Schnitzbauer AA, et al. Prediction of Mortality After ALPPS Stage-1: An Analysis of 320 Patients From the International ALPPS Registry. Ann Surg 2015;262:780-5; discussion 785-6.

7. Serenari M, Collaud C, Alvarez FA, et al. Interstage Assessment of Remnant Liver Function in ALPPS Using Hepatobiliary Scintigraphy: Prediction of Posthepatectomy Liver Failure and Introduction of the HIBA Index. Ann Surg 2018;267:1141-7.

8. Tomassini F, Giglio MC, De Simone G, et al. Hepatic function assessment to predict post-hepatectomy liver failure: what can we trust? A systematic review. Updates Surg 2020;72:925-38.

9. Adam R, Imai K, Castro Benitez C, et al. Outcome after associating liver partition and portal vein ligation for staged hepatectomy and conventional two-stage hepatectomy for colorectal liver metastases. Br J Surg 2016;103:1521-9.

10. Moris D, Ronnekleiv-Kelly S, Kostakis ID, et al. Operative Results and Oncologic Outcomes of Associating Liver Partition and Portal Vein Ligation for Staged Hepatectomy (ALPPS) Versus Two-Stage Hepatectomy (TSH) in Patients with Unresectable Colorectal Liver Metastases: A Systematic Review and Meta-Analysis. World J Surg 2018;42:806-15. 
11. Linecker M, Björnsson B, Stavrou GA, et al. Risk Adjustment in ALPPS Is Associated With a Dramatic Decrease in Early Mortality and Morbidity. Ann Surg 2017;266:779-86.

12. Zhang L, Yang Z, Zhang S, et al. Conventional Two-Stage Hepatectomy or Associating Liver Partitioning and Portal Vein Ligation for Staged Hepatectomy for Colorectal Liver Metastases? A Systematic Review and Meta-Analysis. Front Oncol 2020;10:1391.

13. Sandström P, Røsok BI, Sparrelid E, et al. ALPPS Improves Resectability Compared With Conventional Two-stage Hepatectomy in Patients With Advanced Colorectal Liver Metastasis: Results From a Scandinavian Multicenter Randomized Controlled Trial (LIGRO Trial). Ann Surg 2018;267:833-40.

Cite this article as: Jonas JP, Petrowsky H. The potential role of associating liver partition and portal vein ligation in the treatment of colorectal liver metastases. HepatoBiliary Surg Nutr 2021;10(6):902-905. doi: 10.21037/hbsn-21-420
14. Hasselgren K, Røsok BI, Larsen PN, et al. ALPPS Improves Survival Compared With TSH in Patients Affected of CRLM: Survival Analysis From the Randomized Controlled Trial LIGRO. Ann Surg 2021;273:442-8.

15. Müller PC, Linecker M, Kirimker EO, et al. Induction of liver hypertrophy for extended liver surgery and partial liver transplantation: State of the art of parenchyma augmentation-assisted liver surgery. Langenbecks Arch Surg 2021. [Epub ahead of print]. doi: 10.1007/s00423021-02148-2.

16. Petrowsky H, Linecker M, Raptis DA, et al. First Longterm Oncologic Results of the ALPPS Procedure in a Large Cohort of Patients With Colorectal Liver Metastases. Ann Surg 2020;272:793-800. 